$\operatorname{Nr} 60$

\author{
A. A. El-Deeb, H. A. Elsennary and Eze R. Nwaeze
}

\title{
GENERALIZED WEIGHTED OSTROWSKI, TRAPEZOID AND GRÜSS TYPE INEQUALITIES ON TIME SCALES
}

\begin{abstract}
In this article, using two parameters, we obtain generalizations of a weighted Ostrowski type inequality and its companion inequalities on an arbitrary time scale for functions whose first delta derivatives are bounded. Our work unifies the continuous and discrete versions and can also be applied to the quantum calculus case.
\end{abstract}

KEY WORDs: Montgomery identity, Ostrowski inequality, Trapezoid inequality, Grüss inequality, time scales.

AMS Mathematics Subject Classification: 26D15, 26E70.

\section{Introduction}

In order to compute the absolute deviation of a differentiable function and its integral mean, Ostrowski [44] established the following sharp integral inequality:

Theorem 1. Let $\phi:[\alpha, \beta] \rightarrow \mathbb{R}$ be a function that is continuous on $[\alpha, \beta]$ and differentiable on $(\alpha, \beta)$. Then for all $x \in[\alpha, \beta]$, we have

$$
\left|\phi(x)-\frac{1}{\beta-\alpha} \int_{\alpha}^{\beta} \phi(t) d t\right| \leq \sup _{\alpha<x<\beta}\left|\phi^{\prime}(x)\right|(\beta-\alpha)\left[\frac{\left(x-\frac{\alpha+\beta}{2}\right)^{2}}{(\beta-\alpha)^{2}}+\frac{1}{4}\right] .
$$

The inequality (1), which is known in the literature as Ostrowski's inequality, can be proved by employing the Montgomery identity [36].

Ostrowski's inequality has many applications in numerical analysis. One of these applications is the error estimation of the approximation in integration.

Over several decades, many extensions and generalizations were done to Ostrowski's inequality, we refer the interested reader to the papers $[28,3$, 
$12,16,17,31,29,30,15,14]$, the books $[36,35]$ and the references cited therein.

The theory of time scales, which has recently received a lot of attention, was initiated by S. Hilger in his PhD thesis [19] in order to unify discrete and continuous analysis [20]. The general idea is to prove a result for a dynamic equation or a dynamic inequality where the domain of the unknown function is a so-called time scale $\mathbb{T}$, which may be an arbitrary closed subset of the real numbers $\mathbb{R}$ see $[11,6]$. The three most popular examples of calculus on time scales are differential calculus, difference calculus, and quantum calculus (see [23]), i.e., when $\mathbb{T}=\mathbb{R}, \mathbb{T}=\mathbb{N}$ and $\mathbb{T}=\overline{q^{\mathbb{Z}}}=\left\{q^{z}: z \in \mathbb{Z}\right\} \cup\{0\}$ where $q>1$. The books on the subject of time scales by Bohner and Peterson [10] summarize and organize much of time scale calculus. During the past decade a number of dynamic inequalities has been established by some authors which are motivated by some applications (see $[1,7,8,21,27$, 13, 22, 25, 26, 40, 9, 4, 46, 2, 49]).

In [8], Bohner and Matthews gave the time scales version of the Montgomery identity as follows:

Theorem 2. Let $\alpha, \beta, \eta, \tau \in \mathbb{T}$, with $\alpha<\beta$ and $\phi:[\alpha, \beta]_{\mathbb{T}} \rightarrow \mathbb{R}$ be a delta differentiable function. Then

$$
\phi(\tau)=\frac{1}{\beta-\alpha} \int_{\alpha}^{\beta} \phi^{\sigma}(\eta) \Delta \eta+\frac{1}{\beta-\alpha} \int_{\alpha}^{\beta} L(\eta, \tau) \phi^{\Delta}(\eta) \Delta \eta,
$$

where

$$
L(\eta, \tau)= \begin{cases}\eta-\alpha, & \eta \in[\alpha, \tau)_{\mathbb{T}}, \\ \eta-\beta, & \eta \in[\tau, \beta]_{\mathbb{T}} .\end{cases}
$$

Using the above result, Bohner and Matthews [8] extended the Ostrowski inequality (1) to time scales. Their result can be written as

Theorem 3. Let $\alpha, \beta, \eta, \tau \in \mathbb{T}, \alpha<\beta$ and $\phi:[\alpha, \beta]_{\mathbb{T}} \rightarrow \mathbb{R}$ be a delta differentiable function. Then for all $\tau \in[\alpha, \beta]_{\mathbb{T}}$, we have

$$
\left|\phi(\tau)-\frac{1}{\beta-\alpha} \int_{\alpha}^{\beta} \phi(\sigma(\eta)) \Delta \eta\right| \leq \frac{M}{\beta-\alpha}\left(h_{2}(\tau, \alpha)+h_{2}(\beta, \tau)\right),
$$

where $h_{2}(t, s)=\int_{s}^{t}(\nu-s) \Delta \nu$ and $M=\sup _{\tau \in(\alpha, \beta)}\left|\phi^{\Delta}(\tau)\right|<\infty$. The inequality (3) is sharp in the sense that the right hand side cannot be replaced by a smaller one.

Liu et al. [24] obtained the following generalization of the Montgomery identity on time scales (2): 
Theorem 4. Let $\alpha, \beta, \eta, \tau \in \mathbb{T}$, with $\alpha<\beta$ and $\phi:[\alpha, \beta]_{\mathbb{T}} \rightarrow \mathbb{R}$ be a delta differentiable function. Then

$$
\begin{aligned}
(1-\lambda) \phi(\tau)+ & \lambda \frac{\phi(\alpha)+\phi(\beta)}{2} \\
& =\frac{1}{\beta-\alpha} \int_{\alpha}^{\beta} \phi^{\sigma}(\eta) \Delta \eta+\frac{1}{\beta-\alpha} \int_{\alpha}^{\beta} L(\eta, \tau) \phi^{\Delta}(\eta) \Delta \eta,
\end{aligned}
$$

for all $\lambda \in[0,1]$, where

$$
L(\eta, \tau)= \begin{cases}\eta-\left(\alpha+\lambda \frac{\beta-\alpha}{2}\right), & \eta \in[\alpha, \tau)_{\mathbb{T}}, \\ \eta-\left(\beta-\lambda \frac{\beta-\alpha}{2}\right), & \eta \in[\tau, \beta]_{\mathbb{T}} .\end{cases}
$$

Also in [24], the authors generalized the dynamic Ostrowski inequality (2) by using the generalized Montgomery identity on time scales (4). Their result can be stated as

Theorem 5. Let $\alpha, \beta, \eta, \tau \in \mathbb{T}, \alpha<\beta$ and $\phi:[\alpha, \beta]_{\mathbb{T}} \rightarrow \mathbb{R}$ be a delta differentiable function. Then for all $\tau \in\left[\alpha+\lambda \frac{\beta-\alpha}{2}, \beta-\lambda \frac{\beta-\alpha}{2}\right]_{\mathbb{T}}$ and $\lambda \in[0,1]$, we have

$$
\begin{aligned}
\mid(1-\lambda) \phi(\tau)+ & \lambda \frac{\phi(\alpha)+\phi(\beta)}{2}-\frac{1}{\beta-\alpha} \int_{\alpha}^{\beta} \phi(\sigma(\eta)) \Delta \eta \mid \\
\leq & \frac{M}{\beta-\alpha}\left(h_{2}\left(\alpha, \alpha+\lambda \frac{\beta-\alpha}{2}\right)+h_{2}\left(\tau, \alpha+\lambda \frac{\beta-\alpha}{2}\right)\right. \\
& \left.+h_{2}\left(\tau, \beta-\lambda \frac{\beta-\alpha}{2}\right)+h_{2}\left(\beta, \beta-\lambda \frac{\beta-\alpha}{2}\right)\right)
\end{aligned}
$$

where $h_{2}(t, s)=\int_{s}^{t}(\nu-s) \Delta \nu$ and $M=\sup _{\tau \in(\alpha, \beta)}\left|\phi^{\Delta}(\tau)\right|<\infty$.

Recently, Nwaeze [39] proved the following generalized weighted Montgomery identity on time scales:

Theorem 6. Let $\alpha, \beta, \eta, \tau \in \mathbb{T}, \alpha<\beta, \phi:[\alpha, \beta]_{\mathbb{T}} \rightarrow \mathbb{R}$ be a delta differentiable function and $\psi$ is a function of $[0,1]$ into $[0,1]$. Furthermore, assume that $u:[\alpha, \beta]_{\mathbb{T}} \rightarrow[0, \infty)_{\mathbb{T}}$ be a nonnegative rd-continuous function and $p:[\alpha, \beta]_{\mathbb{T}} \rightarrow \mathbb{R}$ be a delta differentiable function such that $p^{\Delta} \equiv u$ on $[\alpha, \beta]_{\mathbb{T}}$. Then for all $\tau \in[\alpha, \beta]_{\mathbb{T}}$, we have

$$
\begin{aligned}
& \frac{1+\psi(1-\lambda)-\psi(\lambda)}{2} \phi(\tau)+\frac{\psi(\lambda) \phi(\alpha)+(1-\psi(1-\lambda)) \phi(\beta)}{2} \\
& =\frac{1}{\int_{\alpha}^{\beta} u(\eta) \Delta \eta} \int_{\alpha}^{\beta} L(\eta, \tau) \phi^{\Delta}(\eta) \Delta \eta+\frac{1}{\int_{\alpha}^{\beta} u(\eta) \Delta \eta} \int_{\alpha}^{\beta} u(\eta) \phi^{\sigma}(\eta) \Delta \eta
\end{aligned}
$$


where

$$
L(\eta, \tau)= \begin{cases}p(\eta)-\left(p(\alpha)+\psi(\lambda) \frac{p(\beta)-p(\alpha)}{2}\right), & \eta \in[\alpha, \tau)_{\mathbb{T}} \\ p(\eta)-\left(p(\alpha)+(1+\psi(1-\lambda)) \frac{p(\beta)-p(\alpha)}{2}\right), & \eta \in[\tau, \beta]_{\mathbb{T}} .\end{cases}
$$

Nwaeze [39] employed the generalized weighted Montgomery identity (6) to obtain the following weighted Ostrowski type inequality on time scales:

Theorem 7. Let the assumptions of Theorem 6 hold. Then for all $\tau \in[\alpha, \beta]_{\mathbb{T}}$, we have

$$
\begin{array}{r}
\mid\left\{\frac{1+\psi(1-\lambda)-\psi(\lambda)}{2} \phi(\tau)+\frac{\psi(\lambda) \phi(\alpha)+(1-\psi(1-\lambda)) \phi(\beta)}{2}\right\} \\
\quad \times \int_{\alpha}^{\beta} u(\eta) \Delta \eta-\int_{\alpha}^{\beta} u(\eta) \phi^{\sigma}(\eta) \Delta \eta\left|\leq M \int_{\alpha}^{\beta}\right| L(\eta, \tau) \mid \Delta \eta,
\end{array}
$$

where

$$
L(\eta, \tau)= \begin{cases}p(\eta)-\left(p(\alpha)+\psi(\lambda) \frac{p(\beta)-p(\alpha)}{2}\right), & \eta \in[\alpha, \tau)_{\mathbb{T}} \\ p(\eta)-\left(p(\alpha)+(1+\psi(1-\lambda)) \frac{p(\beta)-p(\alpha)}{2}\right), & \eta \in[\tau, \beta]_{\mathbb{T}}\end{cases}
$$

and

$$
M=\sup _{\alpha<\eta<\beta}\left|\phi^{\Delta}(\eta)\right|<\infty .
$$

Some various generalizations and extensions of the dynamic Ostrowski inequality can be found in the papers $[34,33,5,18,50,24,32,37,45,47$, 48, 43, 42, 41].

In this paper, inspired by the above works, we will first give a generalization of the Montgomery identity on time scales by introducing two parameters and then use it to prove new generalized weighted Ostrowski, Trapezoid and Grüss type inequalities on time scales. As special cases of our results, some continuous and discrete inequalities will be stated.

This paper is organized in the following manner: in Section 2, some basic concepts of the calculus on time scales and useful lemmas are recalled. In Section 3, we state and prove the main results.

\section{Preliminaries and lemmas}

First we will present some preliminaries on calculus of time scales and some universal symbols used in this article. Throughout the paper $\mathbb{R}$ denotes the set of real numbers and $\mathbb{Z}$ denotes the set of integers.

A time scale $\mathbb{T}$ is an arbitrary nonempty closed subset of the real numbers. We also assume throughout that $\mathbb{T}$ has the topology that it inherits from the 
standard topology on the real numbers $\mathbb{R}$. For $t \in \mathbb{T}$, we define the forward jump operator $\sigma: \mathbb{T} \rightarrow \mathbb{T}$ by:

$$
\sigma(t):=\inf \{s \in \mathbb{T}: s>t\}
$$

where $\inf \emptyset=\sup \mathbb{T}$ and the backward jump operator $\rho: \mathbb{T} \rightarrow \mathbb{T}$ by:

$$
\rho(t):=\sup \{s \in \mathbb{T}: s<t\}
$$

where $\sup \emptyset=\inf \mathbb{T}$. The jump operators $\sigma$ and $\rho$ allow the classification of a point $t \in \mathbb{T}$ in this manner: if $\sigma(t)>t$, we say that $t$ is right-scattered, while if $\rho(t)<t$ we say that $t$ is left-scattered. Points that are right-scattered and left-scattered at the same time are called isolated. Also, if $t<\sup \mathbb{T}$ and $\sigma(t)=t$, then $t$ is called right-dense, and if $t>\inf \mathbb{T}$ and $\rho(t)=t$, then $t$ is called left-dense. Points that are right-dense and left-dense at the same time are called dense. A function $g: \mathbb{T} \rightarrow \mathbb{R}$ is said to be right-dense continuous ( $\mathrm{rd}$-continuous) provided $g$ is continuous at right-dense points and at left-dense points in $\mathbb{T}$, left hand limits exist and are finite. The set of all such $\mathrm{rd}$-continuous functions is denoted by $C_{r d}(\mathbb{T})$. A function $f: \mathbb{T} \rightarrow \mathbb{R}$ is said to be left-dense continuous (ld-continuous) provided $f$ is continuous at left-dense points and at right-dense points in $\mathbb{T}$, right-hand limits exist and are finite. The set of all such ld-continuous functions is denoted by $C_{l d}(\mathbb{T})$.

The forward and backward graininess functions $\mu$ and $\nu$ for a time scale $\mathbb{T}$ is defined by $\mu(t):=\sigma(t)-t$, and $\nu(t)=t-\rho(t)$, respectively.

Given a time scale $\mathbb{T}$, we introduce the sets $\mathbb{T}^{\kappa}, \mathbb{T}_{\kappa}$, and $\mathbb{T}_{\kappa}^{\kappa}$ as follows. If $\mathbb{T}$ has a left-scattered maximum $t_{1}$, then $\mathbb{T}^{\kappa}=\mathbb{T}-\left\{t_{1}\right\}$, otherwise $\mathbb{T}^{\kappa}=\mathbb{T}$. If $\mathbb{T}$ has a right-scattered minimum $t_{2}$, then $\mathbb{T}_{\kappa}=\mathbb{T}-\left\{t_{2}\right\}$, otherwise $\mathbb{T}^{\kappa}=\mathbb{T}$. Finally $\mathbb{T}_{\kappa}^{k}=\mathbb{T}^{\kappa} \cap \mathbb{T}_{\kappa}$.

Let $f: \mathbb{T} \rightarrow \mathbb{R}$ be a real valued function on a time scale $\mathbb{T}$. Then $\forall t \in \mathbb{T}^{\kappa}$, we define $f^{\Delta}(t)$ to be the number (if it exists) with the property that given any $\varepsilon>0$ there is a neighborhood $U$ of $t$ such that

$$
\left|[f(\sigma(t))-f(s)]-f^{\Delta}(t)[\sigma(t)-s]\right| \leq \varepsilon|\sigma(t)-s|, \quad \forall s \in U .
$$

In this case we say that $f$ is delta differentiable on $\mathbb{T}^{\kappa}$ provided $f^{\Delta}(t)$ exists for all $t \in \mathbb{T}_{\kappa}$. Similarly, $\forall t \in \mathbb{T}_{\kappa}$, we define $f^{\nabla}(t)$ to be the number (if it exists) with the property that given any $\varepsilon>0$ there is a neighborhood $U$ of $t$ such that

$$
\left|[f(\rho(t))-f(s)]-f^{\nabla}(t)[\rho(t)-s]\right| \leq \varepsilon|\rho(t)-s|, \quad \forall s \in U .
$$

In this case we say that $f$ is nabla differentiable on $\mathbb{T}_{\kappa}$ provided $f^{\nabla}(t)$ exists for all $t \in \mathbb{T}_{\kappa}$. For $f: \mathbb{T} \rightarrow \mathbb{R}$, we define the function $f^{\sigma}: \mathbb{T} \rightarrow \mathbb{R}$ 
by $f^{\sigma}(t)=f(\sigma(t)), \forall t \in \mathbb{T}$, that is $f^{\sigma}=f \circ \sigma$. Similarly, we define the function $f^{\rho}: \mathbb{T} \rightarrow \mathbb{R}$ by $f^{\rho}(t)=f(\rho(t)), \forall t \in \mathbb{T}$, that is $f^{\rho}=f \circ \rho$. A time scale $\mathbb{T}$ is said to be regular if the following two conditions are satisfied simultaneously: $(1) \sigma(\rho(t))=t$, and (2) $\rho(\sigma(t))=t, \forall t \in \mathbb{T}$. The product and the quotient rules for the derivative of the product $f g$ and the quotient $f / g$ (where $g g^{\sigma} \neq 0$, here $g^{\sigma}=g \circ \sigma$ ) of two differentiable functions $f$ and $g$, are given as the following:

$$
(f g)^{\Delta}(t)=f^{\Delta}(t) g(t)+f(\sigma(t)) g^{\Delta}(t)=f(t) g^{\Delta}(t)+f^{\Delta}(t) g(\sigma(t)),
$$

and

$$
\left(\frac{f}{g}\right)^{\Delta}(t)=\frac{f^{\Delta}(t) g(t)-f(t) g^{\Delta}(t)}{g(t) g(\sigma(t))} .
$$

A function $F: \mathbb{T} \rightarrow \mathbb{R}$, is called a delta antiderivative of $f: \mathbb{T} \rightarrow \mathbb{R}$ provided that $F^{\Delta}=f(t)$ holds $\forall t \in \mathbb{T}^{\kappa}$, then the delta integral of $f$ is defined by

$$
\int_{a}^{b} f(t) \Delta t=F(b)-F(a) .
$$

A function $G: \mathbb{T} \rightarrow \mathbb{R}$, is called a nabla antiderivative of $g: \mathbb{T} \rightarrow \mathbb{R}$ provided that $G^{\nabla}=g(t)$ holds $\forall t \in \mathbb{T}_{\kappa}$, then the delta integral of $g$ is defined by

$$
\int_{a}^{b} g(t) \nabla t=G(b)-G(a) .
$$

Using (9), Integration by parts formula on time scales is given by

$$
\int_{a}^{b} f^{\Delta}(t) g(t) \Delta t=f(b) g(b)-f(a) g(a)-\int_{a}^{b} f^{\sigma}(t) g^{\Delta}(t) \Delta t .
$$

We will frequently use the following useful relations between calculus on time scales $\mathbb{T}$ and differential calculus on $\mathbb{R}$, difference calculus on $\mathbb{Z}$, and quantum calculus on $\overline{q^{\mathbb{Z}}}$. Note that:

(i) if $\mathbb{T}=\mathbb{R}$, then

$$
\sigma(t)=t, \mu(t)=0, f^{\Delta}(t)=f^{\prime}(t), \int_{a}^{b} f(t) \Delta t=\int_{a}^{b} f(t) d t .
$$

(ii) If $\mathbb{T}=\mathbb{Z}$, then

$$
\sigma(t)=t+1, \mu(t)=1, f^{\Delta}(t)=\Delta f(t), \int_{a}^{b} f(t) \Delta t=\sum_{t=a}^{b-1} f(t) .
$$


(iii) If $\mathbb{T}=h \mathbb{Z}, h>0$, then

$$
\sigma(t)=t+h, \mu(t)=h, \quad \int_{a}^{b} f(t) \Delta t=\sum_{k=0}^{\frac{b-a-h}{h}} f(a+k h) h .
$$

(iv) If $\mathbb{T}=\overline{q^{\mathbb{Z}}}=\left\{q^{z}: z \in \mathbb{Z}\right\} \cup\{0\}, q>1$, then

$$
\sigma(t)=q t, \mu(t)=(q-1) t, \int_{a}^{b} f(t) \Delta t=(q-1) \sum_{k=\log _{q}(a)}^{\log _{q}(b)-1} q^{k} f\left(q^{k}\right),
$$

$\forall a, b \in q^{\mathbb{N}_{0}}$. It can be shown (see [10]) that if $g \in C_{r d}(\mathbb{T})$, then the Cauchy integral $G(t):=\int_{t_{0}}^{t} g(s) \Delta s$ exists, $t_{0} \in \mathbb{T}$, and satisfies $G^{\Delta}(t)=g(t), t \in \mathbb{T}$.

Now we are ready to state and prove our main results.

\section{Main results}

\subsection{A generalized weighted Montgomery identity} on time scales

Lemma 1. Let $\alpha, \beta, \tau, \eta \in \mathbb{T}$ with $\alpha<\beta$ and $\phi:[\alpha, \beta]_{\mathbb{T}} \rightarrow \mathbb{R}$ be a delta differentiable function. Furthermore, assume that $u:[\alpha, \beta]_{\mathbb{T}} \rightarrow[0, \infty)_{\mathbb{T}}$ be a nonnegative rd-continuous function and $p:[\alpha, \beta]_{\mathbb{T}} \rightarrow \mathbb{R}$ be a delta differentiable function such that $p^{\Delta} \equiv u$ on $[\alpha, \beta]_{\mathbb{T}}$. Then for all $\tau \in[\alpha, \beta]_{\mathbb{T}}$ and $\gamma_{1}, \gamma_{2} \in[0,1]$, we have

$$
\begin{aligned}
& \left(1-\gamma_{1}-\gamma_{2}\right) \phi(\tau)+\gamma_{1} \phi(\alpha)+\gamma_{2} \phi(\beta) \\
& \quad=\frac{1}{\int_{\alpha}^{\beta} u(\eta) \Delta \eta} \int_{\alpha}^{\beta} L(\eta, \tau) \phi^{\Delta}(\eta) \Delta \eta+\frac{1}{\int_{\alpha}^{\beta} u(\eta) \Delta \eta} \int_{\alpha}^{\beta} u(\eta) \phi^{\sigma}(\eta) \Delta \eta,
\end{aligned}
$$

where

$$
L(\eta, \tau)= \begin{cases}p(\eta)-\left(p(\alpha)+\gamma_{1}[p(\beta)-p(\alpha)]\right), & \eta \in[\alpha, \tau)_{\mathbb{T}}, \\ p(\eta)-\left(p(\beta)-\gamma_{2}[p(\beta)-p(\alpha)]\right), & \eta \in[\tau, \beta]_{\mathbb{T}} .\end{cases}
$$

Proof. Using integration by parts formula on time scales given by (10), we have

$$
\begin{aligned}
\int_{\alpha}^{\tau} L(\eta, \tau) \phi^{\Delta}(\eta) \Delta \eta \\
=\int_{\alpha}^{\tau}\left[p(\eta)-\left(p(\alpha)+\gamma_{1}[p(\beta)-p(\alpha)]\right)\right] \phi^{\Delta}(\eta) \Delta \eta \\
=\left[p(\tau)-\left(p(\alpha)+\gamma_{1}[p(\beta)-p(\alpha)]\right)\right] \phi(\tau) \\
\quad-\left[p(\alpha)-\left(p(\alpha)+\gamma_{1}[p(\beta)-p(\alpha)]\right)\right] \phi(\alpha)-\int_{\alpha}^{\tau} u(\eta) \phi^{\sigma}(\eta) \Delta \eta
\end{aligned}
$$


and

$$
\begin{aligned}
\int_{\tau}^{\beta} L(\eta, \tau) \phi^{\Delta}(\eta) \Delta \eta \\
=\int_{\tau}^{\beta}\left[p(\eta)-\left(p(\beta)-\gamma_{2}[p(\beta)-p(\alpha)]\right)\right] \phi^{\Delta}(\eta) \Delta \eta \\
=\left[p(\beta)-\left(p(\beta)-\gamma_{2}[p(\beta)-p(\alpha)]\right)\right] \phi(\beta) \\
\quad-\left[p(\tau)-\left(p(\beta)-\gamma_{2}[p(\beta)-p(\alpha)]\right)\right] \phi(\tau)-\int_{\tau}^{\beta} u(\eta) \phi^{\sigma}(\eta) \Delta \eta
\end{aligned}
$$

Adding (15) and (16), we obtain

$$
\begin{aligned}
\int_{\alpha}^{\beta} L(\eta, \tau) \phi^{\Delta}(\eta) \Delta \eta= & \left\{\left(1-\gamma_{1}-\gamma_{2}\right) \phi(\tau)+\gamma_{1} \phi(\alpha)+\gamma_{2} \phi(\beta)\right\} \\
& \times \int_{\alpha}^{\beta} u(\eta) \Delta \eta-\int_{\alpha}^{\beta} u(\eta) \phi^{\sigma}(\eta) \Delta \eta .
\end{aligned}
$$

This gives us the desired identity (15).

Corollary 1. As a special case of Lemma 1 , when $\mathbb{T}=\mathbb{R}$ and using the relation (11), we get

$$
\begin{aligned}
& \left(1-\gamma_{1}-\gamma_{2}\right) \phi(\tau)+\gamma_{1} \phi(\alpha)+\gamma_{2} \phi(\beta) \\
& \quad=\frac{1}{\int_{\alpha}^{\beta} u(\eta) d \eta} \int_{\alpha}^{\beta} L(\eta, \tau) \phi^{\prime}(\eta) d \eta+\frac{1}{\int_{\alpha}^{\beta} u(\eta) d \eta} \int_{\alpha}^{\beta} u(\eta) \phi(\eta) d \eta,
\end{aligned}
$$

where $u(\tau)=p^{\prime}(\tau)$ on $[\alpha, \beta]$ and

$$
L(\eta, \tau)= \begin{cases}p(\eta)-\left(p(\alpha)+\gamma_{1}[p(\beta)-p(\alpha)]\right), & \eta \in[\alpha, \tau), \\ p(\eta)-\left(p(\beta)-\gamma_{2}[p(\beta)-p(\alpha)]\right), & \eta \in[\tau, \beta] .\end{cases}
$$

Corollary 2. As a special case of Lemma 1 , when $\mathbb{T}=\mathbb{Z}$ and using the relation (12), we get

$$
\begin{aligned}
& \left(1-\gamma_{1}-\gamma_{2}\right) \phi(m)+\gamma_{1} \phi(\alpha)+\gamma_{2} \phi(\beta) \\
& \quad=\frac{1}{\sum_{n=\alpha}^{\beta-1} u(n)} \sum_{n=\alpha}^{\beta-1} L(n, m) \Delta \phi(n)+\frac{1}{\sum_{n=\alpha}^{\beta-1} u(n)} \sum_{n=\alpha}^{\beta-1} u(n) \phi(n+1),
\end{aligned}
$$

where $u(n)=\Delta p(n)=p(n+1)-p(n)$ for $n=\alpha, \ldots, \beta-1$ and

$$
L(n, m)= \begin{cases}p(n)-\left(p(\alpha)+\gamma_{1}[p(\beta)-p(\alpha)]\right), & \text { if } n=\alpha, \ldots, m-1, \\ p(n)-\left(p(\beta)-\gamma_{2}[p(\beta)-p(\alpha)]\right), & \text { if } n=m, \ldots, \beta\end{cases}
$$


Corollary 3. If we take $\gamma_{1}=\psi(\lambda) / 2$ and $\gamma_{2}=1-\psi(1-\lambda) / 2$, where $\psi$ is a function from $[0,1]$ into $[0,1]$, in Lemma 1 , we get

$$
\begin{aligned}
& \frac{1+\psi(1-\lambda)-\psi(\lambda)}{2} \phi(\tau)+\frac{\psi(\lambda) \phi(\alpha)+(1-\psi(1-\lambda)) \phi(\beta)}{2} \\
& =\frac{1}{\int_{\alpha}^{\beta} u(\eta) \Delta \eta} \int_{\alpha}^{\beta} L(\eta, \tau) \phi^{\Delta}(\eta) \Delta \eta+\frac{1}{\int_{\alpha}^{\beta} u(\eta) \Delta \eta} \int_{\alpha}^{\beta} u(\eta) \phi^{\sigma}(\eta) \Delta \eta,
\end{aligned}
$$

where

$$
L(\eta, \tau)= \begin{cases}p(\eta)-\left(p(\alpha)+\psi(\lambda) \frac{p(\beta)-p(\alpha)}{2}\right), & \eta \in[\alpha, \tau)_{\mathbb{T}}, \\ p(\eta)-\left(p(\alpha)+(1+\psi(1-\lambda)) \frac{p(\beta)-p(\alpha)}{2}\right), & \eta \in[\tau, \beta]_{\mathbb{T}},\end{cases}
$$

which is exactly the result obtained by Nwaeze in [39].

Corollary 4. If we take $p(\tau)=\tau$ and $\gamma_{1}=\gamma_{2}=0$ in Lemma 1, we get

$$
\phi(\tau)=\frac{1}{\beta-\alpha} \int_{\alpha}^{\beta} L(\eta, \tau) \phi^{\Delta}(\eta) \Delta \eta+\frac{1}{\beta-\alpha} \int_{\alpha}^{\beta} \phi^{\sigma}(\eta) \Delta \eta,
$$

where

$$
L(\eta, \tau)= \begin{cases}\eta-\alpha, & \eta \in[\alpha, \tau)_{\mathbb{T}}, \\ \eta-\beta, & \eta \in[\tau, \beta]_{\mathbb{T}},\end{cases}
$$

which is the Montgomery identity on time scales due to Bohner and Matthews in [8].

\subsection{A generalized weighted Ostrowski inequality on time scales}

Theorem 8. Under the same assumptions as in Lemma 1, we have

$$
\begin{aligned}
& \mid\left\{\left(1-\gamma_{1}-\gamma_{2}\right) \phi(\tau)+\gamma_{1} \phi(\alpha)+\gamma_{2} \phi(\beta)\right\} \\
& \quad \times \int_{\alpha}^{\beta} u(\eta) \Delta \eta-\int_{\alpha}^{\beta} u(\eta) \phi^{\sigma}(\eta) \Delta \eta\left|\leq M \int_{\alpha}^{\beta}\right| L(\eta, \tau) \mid \Delta \eta,
\end{aligned}
$$

where

$$
L(\eta, \tau)= \begin{cases}p(\eta)-\left(p(\alpha)+\gamma_{1}[p(\beta)-p(\alpha)]\right), & \eta \in[\alpha, \tau)_{\mathbb{T}}, \\ p(\eta)-\left(p(\beta)-\gamma_{2}[p(\beta)-p(\alpha)]\right), & \eta \in[\tau, \beta]_{\mathbb{T}},\end{cases}
$$

and

$$
M=\sup _{\alpha<\eta<\beta}\left|\phi^{\Delta}(\eta)\right|<\infty .
$$


Proof. The proof can be easily done using Lemma 1 and modulus properties.

Corollary 5. As a special case of Theorem 8 , when $\mathbb{T}=\mathbb{R}$ and using the relation (11), we get

$$
\begin{aligned}
& \mid\left\{\left(1-\gamma_{1}-\gamma_{2}\right) \phi(\tau)+\gamma_{1} \phi(\alpha)+\gamma_{2} \phi(\beta)\right\} \\
& \quad \times \int_{\alpha}^{\beta} u(\eta) d \eta-\int_{\alpha}^{\beta} u(\eta) \phi(\eta) d \eta\left|\leq M \int_{\alpha}^{\beta}\right| L(\eta, \tau) \mid d \eta,
\end{aligned}
$$

where

$$
L(\eta, \tau)= \begin{cases}p(\eta)-\left(p(\alpha)+\gamma_{1}[p(\beta)-p(\alpha)]\right), & \eta \in[\alpha, \tau), \\ p(\eta)-\left(p(\beta)-\gamma_{2}[p(\beta)-p(\alpha)]\right), & \eta \in[\tau, \beta]\end{cases}
$$

and

$$
M=\sup _{\alpha<\eta<\beta}\left|\phi^{\prime}(\eta)\right|<\infty .
$$

Corollary 6. As a special case of Theorem 8 , when $\mathbb{T}=\mathbb{Z}$ and using the relation (12), we get

$$
\begin{aligned}
& \mid\left\{\left(1-\gamma_{1}-\gamma_{2}\right) \phi(m)+\gamma_{1} \phi(\alpha)+\gamma_{2} \phi(\beta)\right\} \\
& \quad \times \sum_{n=\alpha}^{\beta-1} u(n)-\sum_{n=\alpha}^{\beta-1} u(n) \phi(n)\left|\leq M \sum_{n=\alpha}^{\beta-1}\right| L(n, m) \mid,
\end{aligned}
$$

where

$$
L(n, m)= \begin{cases}p(n)-\left(p(\alpha)+\gamma_{1}[p(\beta)-p(\alpha)]\right), & \text { if } n=\alpha, \ldots, m-1, \\ p(n)-\left(p(\beta)-\gamma_{2}[p(\beta)-p(\alpha)]\right), & \text { if } n=m, \ldots, \beta\end{cases}
$$

and

$$
M=\max _{\alpha<n<\beta}|\Delta \phi(n)|<\infty .
$$

Corollary 7. If we take $\gamma_{1}=\psi(\lambda) / 2$ and $\gamma_{2}=1-\psi(1-\lambda) / 2$, where $\psi$ is a function from $[0,1]$ into $[0,1]$, in Theorem 8 , we get

$$
\begin{gathered}
\mid\left\{\frac{1+\psi(1-\lambda)-\psi(\lambda)}{2} \phi(\tau)+\frac{\psi(\lambda) f(a)+(1-\psi(1-\lambda)) \phi(\beta)}{2}\right\} \\
\times \int_{\alpha}^{\beta} u(\eta) \Delta \eta-\int_{\alpha}^{\beta} u(\eta) \phi^{\sigma}(\eta) \Delta \eta\left|\leq M \int_{\alpha}^{\beta}\right| L(\eta, \tau) \mid \Delta \tau
\end{gathered}
$$


where

$$
L(\eta, \tau)= \begin{cases}p(\eta)-\left(p(\alpha)+\psi(\lambda) \frac{p(\beta)-p(\alpha)}{2}\right), & \eta \in[\alpha, \tau)_{\mathbb{T}} \\ p(\eta)-\left(p(\alpha)+(1+\psi(1-\lambda)) \frac{p(\beta)-p(\alpha)}{2}\right), & \eta \in[\tau, \beta]_{\mathbb{T}}\end{cases}
$$

and

$$
M=\sup _{\alpha<\eta<\beta}\left|\phi^{\Delta}(\eta)\right|<\infty,
$$

which is the result obtained by Nwaeze in [39].

Corollary 8. If we take $p(\tau)=\tau$ and $\gamma_{1}=\gamma_{2}=0$ in Theorem 8, we get

$$
\left|\phi(\tau)-\frac{1}{\beta-\alpha} \int_{\alpha}^{\beta} \phi^{\sigma}(\eta) \Delta \eta\right| \leq \frac{M}{\beta-\alpha}\left(h_{2}(\tau, \alpha)+h_{2}(\beta, \tau)\right),
$$

where

$$
L(\eta, \tau)= \begin{cases}\eta-\alpha, & \eta \in[\alpha, \tau)_{\mathbb{T}}, \\ \eta-\beta, & \eta \in[\tau, \beta]_{\mathbb{T}},\end{cases}
$$

which is the classical Ostrowski inequality on time scales due to Bohner and Matthews in [8].

\subsection{A generalized weighted Trapezoid inequality on time scales}

Theorem 9. Under the same assumptions as in Lemma 1, we have

$$
\begin{aligned}
& \mid\left(1-\gamma_{1}-\gamma_{2}\right)\left[\phi^{2}(\beta)-\phi^{2}(\alpha)\right] \\
& \quad+\left\{\gamma_{1}[\phi(\alpha)+\phi(\sigma(\alpha))]+\gamma_{2}[\phi(\beta)+\phi(\sigma(\beta))]\right\}(\phi(\beta)-\phi(\alpha)) \\
& \quad-\frac{\phi(\beta)-\phi(\alpha)}{\int_{\alpha}^{\beta} u(\eta) \Delta \eta} \int_{\alpha}^{\beta} u(\eta)\left[\phi(\sigma(\eta))+\phi\left(\sigma^{2}(\eta)\right)\right] \Delta \eta \mid \\
& \leq \frac{M(M+N)}{\int_{\alpha}^{\beta} u(\eta) \Delta \eta} \int_{\alpha}^{\beta}\left(\int_{\alpha}^{\beta}|L(\eta, \tau)| \Delta \eta\right) \Delta \tau,
\end{aligned}
$$

where

$$
L(\eta, \tau)= \begin{cases}p(\eta)-\left(p(\alpha)+\gamma_{1}[p(\beta)-p(\alpha)]\right), & \eta \in[\alpha, \tau)_{\mathbb{T}}, \\ p(\eta)-\left(p(\beta)-\gamma_{2}[p(\beta)-p(\alpha)]\right), & \eta \in[\tau, \beta]_{\mathbb{T}}\end{cases}
$$

and

$$
M=\sup _{\alpha<\eta<\beta}\left|\phi^{\Delta}(\eta)\right|<\infty \quad \text { and } \quad N=\sup _{\alpha<\eta<\beta}\left|\phi^{\Delta}(\sigma(\eta))\right|<\infty \text {. }
$$


Proof. From Lemma 1, we have

(18)

$$
\begin{aligned}
& \left(1-\gamma_{1}-\gamma_{2}\right) \phi(\tau)=-\gamma_{1} \phi(\alpha)-\gamma_{2} \phi(\beta) \\
& +\frac{1}{\int_{\alpha}^{\beta} u(\eta) \Delta \eta} \int_{\alpha}^{\beta} L(\eta, \tau) \phi^{\Delta}(\eta) \Delta \eta+\frac{1}{\int_{\alpha}^{\beta} u(\eta) \Delta \eta} \int_{\alpha}^{\beta} u(\eta) \phi(\sigma(\eta)) \Delta \eta
\end{aligned}
$$

and

$$
\begin{aligned}
& \left(1-\gamma_{1}-\gamma_{2}\right) \phi(\sigma(\tau))=-\gamma_{1} \phi(\sigma(\alpha))-\gamma_{2} \phi(\sigma(\beta))+\frac{1}{\int_{\alpha}^{\beta} u(\eta) \Delta \eta} \\
& \times \int_{\alpha}^{\beta} L(\eta, \tau) \phi^{\Delta}(\sigma(\eta)) \Delta \eta+\frac{1}{\int_{\alpha}^{\beta} u(\eta) \Delta \eta} \int_{\alpha}^{\beta} u(\eta) \phi\left(\sigma^{2}(\eta)\right) \Delta \eta .
\end{aligned}
$$

Adding (18) and (19), we get

$$
\begin{aligned}
\left(1-\gamma_{1}-\gamma_{2}\right)[\phi(\tau)+\phi(\sigma(\tau))] & \\
= & -\gamma_{1}[\phi(\alpha)+\phi(\sigma(\alpha))]-\gamma_{2}[\phi(\beta)+\phi(\sigma(\beta))] \\
& +\frac{1}{\int_{\alpha}^{\beta} u(\eta) \Delta \eta} \int_{\alpha}^{\beta} L(\eta, \tau)\left[\phi^{\Delta}(\eta)+\phi^{\Delta}(\sigma(\eta))\right] \Delta \eta \\
& +\frac{1}{\int_{\alpha}^{\beta} u(\eta) \Delta \eta} \int_{\alpha}^{\beta} u(\eta)\left[\phi(\sigma(\eta))+\phi\left(\sigma^{2}(\eta)\right) \Delta \eta .\right.
\end{aligned}
$$

Multiplying the last identity by $\phi^{\Delta}(\tau)$, using (9) and integrating the resulting identity with respect to $\tau$ from $\alpha$ to $\beta$ yield

$$
\begin{aligned}
\left(1-\gamma_{1}-\gamma_{2}\right)\left[\phi^{2}(\beta)-\phi^{2}(\alpha)\right] \\
=\left\{-\gamma_{1}[\phi(\alpha)+\phi(\sigma(\alpha))]-\gamma_{2}[\phi(\beta)+\phi(\sigma(\beta))]\right\}(\phi(\beta)-\phi(\alpha)) \\
\quad+\frac{\phi(\beta)-\phi(\alpha)}{\int_{\alpha}^{\beta} u(\eta) \Delta \eta} \int_{\alpha}^{\beta} u(\eta)\left[\phi(\sigma(\eta))+\phi\left(\sigma^{2}(\eta)\right)\right] \Delta \eta \\
\quad+\frac{1}{\int_{\alpha}^{\beta} u(\eta) \Delta \eta} \int_{\alpha}^{\beta} \phi^{\Delta}(\tau)\left(\int_{\alpha}^{\beta} L(\eta, \tau)\left[\phi^{\Delta}(\eta)+\phi^{\Delta}(\sigma(\eta))\right] \Delta \eta\right) \Delta \tau .
\end{aligned}
$$

Equivalently, we have

$$
\begin{aligned}
\left(1-\gamma_{1}-\right. & \left.\gamma_{2}\right)\left[\phi^{2}(\beta)-\phi^{2}(\alpha)\right] \\
& +\left\{\gamma_{1}[\phi(\alpha)+\phi(\sigma(\alpha))]+\gamma_{2}[\phi(\beta)+\phi(\sigma(\beta))]\right\}(\phi(\beta)-\phi(\alpha)) \\
& -\frac{\phi(\beta)-\phi(\alpha)}{\int_{\alpha}^{\beta} u(\eta) \Delta \eta} \int_{\alpha}^{\beta} u(\eta)\left[\phi(\sigma(\eta))+\phi\left(\sigma^{2}(\eta)\right)\right] \Delta \eta \\
= & \frac{1}{\int_{\alpha}^{\beta} u(\eta) \Delta \eta} \int_{\alpha}^{\beta} \int_{\alpha}^{\beta} \phi^{\Delta}(\tau) L(\eta, \tau)\left[\phi^{\Delta}(\eta)+\phi^{\Delta}(\sigma(\eta))\right] \Delta \eta \Delta \tau .
\end{aligned}
$$


Taking the absolute value of both sides, we get

$$
\begin{aligned}
\mid(1- & \left.\gamma_{1}-\gamma_{2}\right)\left[\phi^{2}(\beta)-\phi^{2}(\alpha)\right] \\
& +\left\{\gamma_{1}[\phi(\alpha)+\phi(\sigma(\alpha))]+\gamma_{2}[\phi(\beta)+\phi(\sigma(\beta))]\right\}(\phi(\beta)-\phi(\alpha)) \\
& -\frac{\phi(\beta)-\phi(\alpha)}{\int_{\alpha}^{\beta} u(\eta) \Delta \eta} \int_{\alpha}^{\beta} u(\eta)\left[\phi(\sigma(\eta))+\phi\left(\sigma^{2}(\eta)\right)\right] \Delta \eta \mid \\
= & \left|\frac{1}{\int_{\alpha}^{\beta} u(\eta) \Delta \eta} \int_{\alpha}^{\beta} \int_{\alpha}^{\beta} \phi^{\Delta}(\tau) L(\eta, \tau)\left[\phi^{\Delta}(\eta)+\phi^{\Delta}(\sigma(\eta))\right] \Delta \eta \Delta \tau\right| \\
\leq & \frac{1}{\int_{\alpha}^{\beta} u(\eta) \Delta \eta} \int_{\alpha}^{\beta} \int_{\alpha}^{\beta}\left|\phi^{\Delta}(\tau)\right||L(\eta, \tau)|\left[\left|\phi^{\Delta}(\eta)\right|+\left|\phi^{\Delta}(\sigma(\eta))\right|\right] \Delta \eta \Delta \tau \\
\leq & \frac{M(M+N)}{\int_{\alpha}^{\beta} u(\eta) \Delta \eta} \int_{\alpha}^{\beta} \int_{\alpha}^{\beta}|L(\eta, \tau)| \Delta \eta \Delta \tau .
\end{aligned}
$$

Hence (17) holds.

Corollary 9. As a special case of Theorem 9, when $\mathbb{T}=\mathbb{R}$ and using the relation (11), we get

$$
\begin{aligned}
& \mid\left(1-\gamma_{1}-\gamma_{2}\right) \frac{\phi^{2}(\beta)-\phi^{2}(\alpha)}{2}+\left\{\gamma_{1} \phi(\alpha)+\gamma_{2} \phi(\beta)\right\}(\phi(\beta)-\phi(\alpha)) \\
& -\frac{\phi(\beta)-\phi(\alpha)}{\int_{\alpha}^{\beta} u(\eta) d \eta} \int_{\alpha}^{\beta} u(\eta) \phi(\eta) d \eta \mid \leq \frac{M^{2}}{\int_{\alpha}^{\beta} u(\eta) d \eta} \int_{\alpha}^{\beta}\left(\int_{\alpha}^{\beta}|L(\eta, \tau)| d \eta\right) d \tau,
\end{aligned}
$$

where

$$
L(\eta, \tau)= \begin{cases}p(\eta)-\left(p(\alpha)+\gamma_{1}[p(\beta)-p(\alpha)]\right), & \eta \in[\alpha, \tau), \\ p(\eta)-\left(p(\beta)-\gamma_{2}[p(\beta)-p(\alpha)]\right), & \eta \in[\tau, \beta]\end{cases}
$$

and

$$
M=\sup _{\alpha<\eta<\beta}\left|\phi^{\prime}(\eta)\right|<\infty .
$$

Corollary 10. As a special case of Theorem 9 , when $\mathbb{T}=\mathbb{Z}$ and using the relation (12), we get

$$
\begin{aligned}
& \mid\left(1-\gamma_{1}-\gamma_{2}\right)\left[\phi^{2}(\beta)-\phi^{2}(\alpha)\right] \\
& \quad+\left\{\gamma_{1}[\phi(\alpha)+\phi(\alpha+1)]+\gamma_{2}[\phi(\beta)+\phi(\beta+1)]\right\}(\phi(\beta)-\phi(\alpha))
\end{aligned}
$$




$$
\begin{aligned}
& -\frac{\phi(\beta)-\phi(\alpha)}{\sum_{n=\alpha}^{\beta-1} u(n)} \sum_{n=\alpha}^{\beta-1} u(n)[\phi(n+1)+\phi(n+2)] \mid \\
\leq & \frac{M(M+N)}{\sum_{n=\alpha}^{\beta-1} u(n)} \sum_{m=\alpha}^{\beta-1}\left(\sum_{n=\alpha}^{\beta-1}|L(n, m)|\right)
\end{aligned}
$$

where

$$
L(n, m)= \begin{cases}p(n)-\left(p(\alpha)+\gamma_{1}[p(\beta)-p(\alpha)]\right), & \text { if } n=\alpha, \ldots, m-1, \\ p(n)-\left(p(\beta)-\gamma_{2}[p(\beta)-p(\alpha)]\right), & \text { if } n=m, \ldots, \beta\end{cases}
$$

and

$$
M=\max _{\alpha<n<\beta}|\Delta \phi(n)|<\infty \quad \text { and } \quad N=\max _{\alpha<n<\beta}|\Delta \phi(n+1)|<\infty .
$$

If we take $\gamma_{1}=\psi(\lambda) / 2$ and $\gamma_{2}=1-\psi(1-\lambda) / 2$ in Theorem 9 , we get

Corollary 11. If we take $\gamma_{1}=\psi(\lambda) / 2$ and $\gamma_{2}=1-\psi(1-\lambda) / 2$ in Theorem 9, we get

$$
\begin{aligned}
& \mid(1+\psi(1-\lambda)-\psi(\lambda)) \frac{\phi^{2}(\beta)-\phi^{2}(\alpha)}{2} \\
& +\left\{\psi(\lambda) \frac{\phi(\alpha)+\phi(\sigma(\alpha))}{2}+(1-\psi(1-\lambda)) \frac{\phi(\beta)+\phi(\sigma(\beta))}{2}\right\} \\
& \quad \times(\phi(\beta)-\phi(\alpha))-\frac{\phi(\beta)-\phi(\alpha)}{\int_{\alpha}^{\beta} u(\eta) \Delta \eta} \int_{\eta}^{\beta} u(\eta)\left[\phi(\sigma(\eta))+\phi\left(\sigma^{2}(\eta)\right)\right] \Delta \eta \mid \\
& \leq \frac{M(M+N)}{\int_{\alpha}^{\beta} u(\eta) \Delta \eta} \int_{\alpha}^{\beta}\left(\int_{\alpha}^{\beta}|L(\eta, \tau)| \Delta \eta\right) \Delta \tau
\end{aligned}
$$

where

$$
L(\eta, \tau)= \begin{cases}p(\eta)-\left(p(\alpha)+\psi(\lambda) \frac{p(\beta)-p(\alpha)}{2}\right), & \eta \in[\alpha, \tau)_{\mathbb{T}}, \\ p(\eta)-\left(p(\alpha)+(1+\psi(1-\lambda)) \frac{p(\beta)-p(\alpha)}{2}\right), & \eta \in[\tau, \beta]_{\mathbb{T}}\end{cases}
$$

and

$$
M=\sup _{\alpha<\eta<\beta}\left|\phi^{\Delta}(\eta)\right|<\infty,
$$

which is the result obtained by Nwaeze in [38].

Corollary 12. If we take $p(\tau)=\tau$ and $\gamma_{1}=\gamma_{2}=0$ in Theorem 9, we get

$$
\begin{aligned}
& \left|\left[\phi^{2}(\beta)-\phi^{2}(\alpha)\right]-\frac{\phi(\beta)-\phi(\alpha)}{\beta-\alpha} \int_{\alpha}^{\beta}\left[\phi(\sigma(\eta))+\phi\left(\sigma^{2}(\eta)\right)\right] \Delta \eta\right| \\
& \leq \frac{M(M+N)}{\beta-\alpha} \int_{\alpha}^{\beta}\left(h_{2}(\tau, \alpha)+h_{2}(\beta, \tau)\right) \Delta \tau,
\end{aligned}
$$


where

$$
L(\eta, \tau)= \begin{cases}\eta-\alpha, & \eta \in[\alpha, \tau)_{\mathbb{T}} \\ \eta-\beta, & \eta \in[\tau, \beta]_{\mathbb{T}}\end{cases}
$$

and

$$
M=\sup _{\alpha<\eta<\beta}\left|\phi^{\Delta}(\eta)\right|<\infty \quad \text { and } \quad N=\sup _{\alpha<\eta<\beta}\left|\phi^{\Delta}(\sigma(\eta))\right|<\infty .
$$

\subsection{A generalized weighted Grüss inequality on time scales}

Theorem 10. Let $\alpha, \beta, \eta, \tau \in \mathbb{T}$ with $\alpha<\beta$ and $f, g:[\alpha, \beta]_{\mathbb{T}} \rightarrow \mathbb{R}$ be delta differentiable functions. Furthermore, assume that $u:[\alpha, \beta]_{\mathbb{T}} \rightarrow$ $[0, \infty)_{\mathbb{T}}$ be a nonnegative rd-continuous function and $p:[\alpha, \beta]_{\mathbb{T}} \rightarrow \mathbb{R}$ be a delta differentiable function such that $p^{\Delta} \equiv u$ on $[\alpha, \beta]_{\mathbb{T}}$. Then for all $\eta \in[\alpha, \beta]_{\mathbb{T}}$ and $\gamma_{1}, \gamma_{2} \in[0,1]$, we have

$$
\begin{aligned}
\mid 2(1- & \left.\left.\gamma_{1}-\gamma_{2}\right)\left(\int_{\alpha}^{\beta} u(\eta) \Delta \eta\right)\right)\left(\int_{\alpha}^{\beta} f(\tau) g(\tau) \Delta \tau\right)+\left(\int_{\alpha}^{\beta} u(\eta) \Delta \eta\right) \\
& \times \int_{\alpha}^{\beta}\left[g(\tau)\left[\gamma_{1} f(\alpha)+\gamma_{2} f(\beta)\right]+f(\tau)\left[\gamma_{1} g(\alpha)+\gamma_{2} g(\beta)\right]\right] \Delta \tau \\
- & {\left[\left(\int_{\alpha}^{\beta} g(\tau) \Delta \tau\right)\left(\int_{\alpha}^{\beta} u(\eta) f(\sigma(\eta)) \Delta \eta\right)\right.} \\
+ & \left.\left(\int_{\alpha}^{\beta} f(\tau) \Delta \tau\right)\left(\int_{\alpha}^{\beta} u(\eta) g(\sigma(\eta)) \Delta \eta\right)\right] \mid \\
\leq & \int_{\alpha}^{\beta}(F|g(\tau)|+G|f(\tau)|)\left(\int_{\alpha}^{\beta}|L(\eta, \tau)| \Delta \eta\right) \Delta \tau
\end{aligned}
$$

where

$$
L(\eta, \tau)= \begin{cases}p(\eta)-\left(p(\alpha)+\gamma_{1}[p(\beta)-p(\alpha)]\right), & \eta \in[\alpha, \tau)_{\mathbb{T}}, \\ p(\eta)-\left(p(\beta)-\gamma_{2}[p(\beta)-p(\alpha)]\right), & \eta \in[\tau, \beta]_{\mathbb{T}}\end{cases}
$$

and

$$
F=\sup _{\alpha<\tau<\beta}\left|f^{\Delta}(\tau)\right|<\infty \quad \text { and } \quad G=\sup _{\alpha<\tau<\beta}\left|g^{\Delta}(\tau)\right|<\infty .
$$

Proof. From Lemma 1, we have

$$
\begin{aligned}
& \left(1-\gamma_{1}-\gamma_{2}\right) f(\tau)=-\gamma_{1} f(\alpha)-\gamma_{2} f(\beta) \\
& \quad+\frac{1}{\int_{\alpha}^{\beta} u(\eta) \Delta \eta} \int_{\alpha}^{\beta} u(\eta) f^{\sigma}(\eta) \Delta \eta+\frac{1}{\int_{\alpha}^{\beta} u(\eta) \Delta \eta} \int_{\alpha}^{\beta} L(\eta, \tau) f^{\Delta}(\eta) \Delta \eta
\end{aligned}
$$


and

$(22)$

$$
\begin{aligned}
& \left(1-\gamma_{1}-\gamma_{2}\right) g(\tau)=-\gamma_{1} g(\alpha)-\gamma_{2} g(\beta) \\
& \quad+\frac{1}{\int_{\alpha}^{\beta} u(\eta) \Delta \eta} \int_{\alpha}^{\beta} u(\eta) g^{\sigma}(\eta) \Delta \eta+\frac{1}{\int_{\alpha}^{\beta} u(\eta) \Delta \eta} \int_{\alpha}^{\beta} L(\eta, \tau) g^{\Delta}(\eta) \Delta \eta .
\end{aligned}
$$

Multiplying (21) by $g(\tau)$ and $(22)$ by $f(\tau)$, adding them and integrating the resulting identity with respect to $\tau$ from $\alpha$ to $\beta$ yield

$$
\begin{aligned}
2(1- & \left.\gamma_{1}-\gamma_{2}\right) \int_{\alpha}^{\beta} f(\tau) g(\tau) \Delta \tau \\
= & -\int_{\alpha}^{\beta}\left[g(\tau)\left[\gamma_{1} f(\alpha)+\gamma_{2} f(\beta)\right]+f(\tau)\left[\gamma_{1} g(\alpha)+\gamma_{2} g(\beta)\right]\right] \Delta \tau \\
& +\frac{1}{\int_{\alpha}^{\beta} u(\eta) \Delta \eta}\left[\left(\int_{\alpha}^{\beta} g(\tau) \Delta \tau\right)\left(\int_{\alpha}^{\beta} u(\eta) f(\sigma(\eta)) \Delta \eta\right)\right. \\
& \left.+\left(\int_{\alpha}^{\beta} f(\tau) \Delta \tau\right)\left(\int_{\alpha}^{\beta} u(\eta) g(\sigma(\eta)) \Delta \eta\right)\right] \\
& +\frac{1}{\int_{\alpha}^{\beta} u(\eta) \Delta \eta}\left[\int_{\alpha}^{\beta} g(\tau)\left(\int_{\alpha}^{\beta} L(\eta, \tau) f^{\Delta}(\eta) \Delta \eta\right) \Delta \tau\right. \\
& \left.+\int_{\alpha}^{\beta} f(\tau)\left(\int_{\alpha}^{\beta} L(\eta, \tau) g^{\Delta}(\eta) \Delta \eta\right) \Delta \tau\right] .
\end{aligned}
$$

Using modulus properties, we obtain

$$
\begin{aligned}
\mid 2(1- & \left.\gamma_{1}-\gamma_{2}\right)\left(\int_{\alpha}^{\beta} u(\eta) \Delta \eta\right)\left(\int_{\alpha}^{\beta} f(\tau) g(\tau) \Delta \tau\right)+\left(\int_{\alpha}^{\beta} u(\eta) \Delta \eta\right) \\
& \times \int_{\alpha}^{\beta}\left[g(\tau)\left[\gamma_{1} f(\alpha)+\gamma_{2} f(\beta)\right]+f(\tau)\left[\gamma_{1} g(\alpha)+\gamma_{2} g(\beta)\right]\right] \Delta \tau \\
- & {\left[\left(\int_{\alpha}^{\beta} g(\tau) \Delta \tau\right)\left(\int_{\alpha}^{\beta} u(\eta) f(\sigma(\eta)) \Delta \eta\right)\right.} \\
+ & \left.\left(\int_{\alpha}^{\beta} f(\tau) \Delta \tau\right)\left(\int_{\alpha}^{\beta} u(\eta) g(\sigma(\eta)) \Delta \eta\right)\right] \mid \\
\leq & \mid \int_{\alpha}^{\beta} g(\tau)\left(\int_{\alpha}^{\beta} L(\eta, \tau) f^{\Delta}(\eta) \Delta \eta\right) \Delta \tau \\
+ & \int_{\alpha}^{\beta} f(\tau)\left(\int_{\alpha}^{\beta} L(\eta, \tau) g^{\Delta}(\eta) \Delta \eta\right) \Delta \tau \mid \\
\leq & \int_{\alpha}^{\beta}|g(\tau)|\left(\int_{\alpha}^{\beta}|L(\eta, \tau)|\left|f^{\Delta}(\eta)\right| \Delta \eta\right) \Delta \tau
\end{aligned}
$$




$$
\begin{aligned}
& +\int_{\alpha}^{\beta}|f(\tau)|\left(\int_{\alpha}^{\beta}|L(\eta, \tau)|\left|g^{\Delta}(\eta)\right| \Delta \eta\right) \Delta \tau \\
\leq & \int_{\alpha}^{\beta}(F|g(\tau)|+G|f(\tau)|)\left(\int_{\alpha}^{\beta}|L(\eta, \tau)| \Delta \eta\right) \Delta \tau .
\end{aligned}
$$

This shows the validity of $(20)$.

Corollary 13. As a special case of Theorem 10, when $\mathbb{T}=\mathbb{R}$ and using the relation (11), we get

$$
\begin{aligned}
\mid 2\left(1-\gamma_{1}\right. & \left.-\gamma_{2}\right)\left(\int_{\alpha}^{\beta} u(\eta) d \eta\right)\left(\int_{\alpha}^{\beta} f(\tau) g(\tau) d \tau\right)+\left(\int_{\alpha}^{\beta} u(\eta) d \eta\right) \\
& \times \int_{\alpha}^{\beta}\left[g(\tau)\left[\gamma_{1} f(\alpha)+\gamma_{2} f(\beta)\right]+f(\tau)\left[\gamma_{1} g(\alpha)+\gamma_{2} g(\beta)\right]\right] d \tau \\
- & {\left[\left(\int_{\alpha}^{\beta} g(\tau) d \tau\right)\left(\int_{\alpha}^{\beta} u(\eta) f(\eta) d \eta\right)\right.} \\
+ & \left.\left(\int_{\alpha}^{\beta} f(\tau) d \tau\right)\left(\int_{\alpha}^{\beta} u(\eta) g(\eta) d \eta\right)\right] \mid \\
\leq & \int_{\alpha}^{\beta}(F|g(\tau)|+G|f(\tau)|)\left(\int_{\alpha}^{\beta}|L(\eta, \tau)| d \eta\right) d \tau
\end{aligned}
$$

where

$$
L(\eta, \tau)= \begin{cases}p(\eta)-\left(p(\alpha)+\gamma_{1}[p(\beta)-p(\alpha)]\right), & \eta \in[\alpha, \tau), \\ p(\eta)-\left(p(\beta)-\gamma_{2}[p(\beta)-p(\alpha)]\right), & \eta \in[\tau, \beta]\end{cases}
$$

and

$$
F=\sup _{\alpha<\tau<\beta}\left|f^{\prime}(\tau)\right|<\infty \quad \text { and } \quad G=\sup _{\alpha<\tau<\beta}\left|g^{\prime}(\tau)\right|<\infty .
$$

Corollary 14. As a special case of Theorem 10 , when $\mathbb{T}=\mathbb{Z}$ and using the relation (12), we get

$$
\begin{aligned}
2\left(1-\gamma_{1}\right. & \left.-\gamma_{2}\right)\left(\sum_{n=\alpha}^{\beta-1} u(n)\right)\left(\sum_{m=\alpha}^{\beta-1} f(m) q(m)\right)+\left(\sum_{n=\alpha}^{\beta-1} u(n)\right) \\
& \left.\left.\times \sum_{m=\alpha}^{\beta-1}\left[g(m)\left[\gamma_{1} f(\alpha)+\gamma_{2} f(\beta)\right)\right]+f(m)\left[\gamma_{1} g(\alpha)+\gamma_{2} g(\beta)\right)\right]\right] \\
- & {\left[\left(\sum_{m=\alpha}^{\beta-1} g(m)\right)\left(\sum_{n=\alpha}^{\beta-1} u(n) f(n+1)\right)\right.} \\
+ & \left.\left(\sum_{m=\alpha}^{\beta-1} f(m)\right)\left(\sum_{n=\alpha}^{\beta-1} u(n) g(n+1)\right)\right] \mid
\end{aligned}
$$




$$
\leq \mid \sum_{m=\alpha}^{\beta-1}(F|g(m)|+G|f(m)|)\left(\sum_{n=\alpha}^{\beta-1}|L(n, m)|\right),
$$

where

$$
L(n, m)= \begin{cases}p(n)-\left(p(\alpha)+\gamma_{1}[p(\beta)-p(\alpha)]\right), & \text { if } n=\alpha, \ldots, m-1, \\ p(n)-\left(p(\beta)-\gamma_{2}[p(\beta)-p(\alpha)]\right), & \text { if } n=m, \ldots, \beta\end{cases}
$$

and

$$
F=\max _{\alpha<m<\beta}|\Delta f(m)|<\infty \quad \text { and } \quad G=\max _{\alpha<m<\beta}|\Delta g(m)|<\infty .
$$

Corollary 15. If we take $\gamma_{1}=\psi(\lambda) / 2$ and $\gamma_{2}=1-\psi(1-\lambda) / 2$ in Theorem 10, we get

$$
\begin{aligned}
\mid(1+\psi & \left.(1-\lambda)-\psi(\lambda))\left(\int_{\alpha}^{\beta} u(\eta) \Delta \eta\right)\right)\left(\int_{\alpha}^{\beta} f(\tau) g(\tau) \Delta \tau\right) \\
+ & \left(\int_{\alpha}^{\beta} u(\eta) \Delta \eta\right) \int_{\alpha}^{\beta}\left[g(\tau)\left(\frac{\psi(\lambda) f(\alpha)+(1-\psi(1-\lambda)) f(\beta)}{2}\right)\right. \\
+ & \left.f(\tau)\left(\frac{\psi(\lambda) g(\alpha)+(1-\psi(1-\lambda)) g(\beta)}{2}\right)\right] \Delta \tau \\
- & {\left[\left(\int_{\alpha}^{\beta} g(\tau) \Delta \tau\right)\left(\int_{\alpha}^{\beta} u(\eta) f(\sigma(\eta)) \Delta \eta\right)\right.} \\
+ & \left.\left(\int_{\alpha}^{\beta} f(\tau) \Delta \tau\right)\left(\int_{\alpha}^{\beta} u(\eta) g(\sigma(\eta)) \Delta \eta\right)\right] \mid \\
\leq & \mid \int_{\alpha}^{\beta}(F|g(\tau)|+G|f(\tau)|)\left(\int_{\alpha}^{\beta}|L(\eta, \tau)| \Delta \eta\right) \Delta \tau,
\end{aligned}
$$

where

$$
L(\eta, \tau)= \begin{cases}p(\eta)-\left(p(\alpha)+\psi(\lambda) \frac{p(\beta)-p(\alpha)}{2}\right), & \eta \in[\alpha, \tau)_{\mathbb{T}}, \\ p(\eta)-\left(p(\alpha)+(1+\psi(1-\lambda)) \frac{p(\beta)-p(\alpha)}{2}\right), & \eta \in[\tau, \beta]_{\mathbb{T}}\end{cases}
$$

and

$$
F=\sup _{\alpha<\tau<\beta}\left|f^{\Delta}(\tau)\right|<\infty \quad \text { and } \quad G=\sup _{\alpha<\tau<\beta}\left|g^{\Delta}(\tau)\right|<\infty,
$$

which is the result obtained by Nwaeze in [38]. 
Corollary 16. If we take $p(\tau)=\tau$ and $\gamma_{1}=\gamma_{2}=0$ in Theorem 10, we get

$$
\begin{aligned}
\mid \frac{1}{\beta-\alpha} & \left(\int_{\alpha}^{\beta} f(\tau) g(\tau) \Delta \tau\right) \\
- & {\left[\frac{1}{2}\left(\frac{1}{\beta-\alpha} \int_{\alpha}^{\beta} g(\tau) \Delta \tau\right)\left(\frac{1}{\beta-\alpha} \int_{\alpha}^{\beta} f(\sigma(\eta)) \Delta \eta\right)\right.} \\
& \left.+\frac{1}{2}\left(\frac{1}{\beta-\alpha} \int_{\alpha}^{\beta} f(\tau) \Delta \tau\right)\left(\frac{1}{\beta-\alpha} \int_{\alpha}^{\beta} g(\sigma(\eta)) \Delta \eta\right)\right] \mid \\
\leq & \frac{1}{2(\beta-\alpha)^{2}} \mid \int_{\alpha}^{\beta}(F|g(\tau)|+G|f(\tau)|)\left(h_{2}(\tau, \alpha)+h_{2}(\beta, \tau)\right) \Delta \tau
\end{aligned}
$$

where

$$
L(\eta, \tau)= \begin{cases}\eta-\alpha, & \eta \in[\alpha, \tau)_{\mathbb{T}}, \\ \eta-\beta, & \eta \in[\tau, \beta]_{\mathbb{T}}\end{cases}
$$

and

$$
F=\sup _{\alpha<\tau<\beta}\left|f^{\Delta}(\tau)\right|<\infty \quad \text { and } \quad G=\sup _{\alpha<\tau<\beta}\left|g^{\Delta}(\tau)\right|<\infty .
$$

Acknowledgement. We appreciate the comments and suggestions of the two anonymous referees.

\section{References}

[1] Agarwal R., Bohner M., Peterson A., Inequalities on time scales: a survey, Mathematical Inequalities and Applications, 4(2001), 535-558.

[2] Agarwal R., O'Regan D., Saker S., Dynamic inequalities on time scales, vol. 2014, Springer, 2014.

[3] Ahmad F., Cerone P., Dragomir S.S., Mir N.A., On some bounds of Ostrowski and Čebyšev type, J. Math. Inequal., 4(1)(2010), 53-65.

[4] Ammi M.R.S., Torres D.F.M., Combined dynamic Grüss inequalities on time scales, Journal of Mathematical Sciences, 161(6)(2009), 792-802.

[5] Anastassiou G.A., Representations and Ostrowski type inequalities on time scales, Computers $\&$ Mathematics with Applications, 62(10)(2011), 3933-3958.

[6] Bohner M., Erbe L., Peterson A., Oscillation for nonlinear second order dynamic equations on a time scale, J. Math. Anal. Appl., 301(2)(2005), 491-507.

[7] Bohner M., Matthews T., The Grüss inequality on time scales, Communications in Mathematical Analysis, 3(1)(2007).

[8] Bohner M., Matthews T., Ostrowski inequalities on time scales, J. Inequal. Pure Appl. Math., 9(1)(2008), pages 8.

[9] Bohner M., Matthews T., Tuna A., Diamond-alpha Grüss type inequalities on time scales, International Journal of Dynamical Systems and Differential Equations, 3(1-2)(2011), 234-247. 
[10] Bohner M., Peterson A., Dynamic equations on time scales, Birkhäuser Boston, Inc., Boston, MA, 2001. An introduction with applications.

[11] Bohner M., Peterson A., Advances in dynamic equations on time scales, Birkhäuser Boston, Inc., Boston, MA, 2003.

[12] Cerone P., Dragomir S.S., Roumeliotis J., An inequality of OstrowskiGrüss type for twice differentiable mappings and applications in numerical integration, RGMIA research report collection, 1(2)(1998).

[13] Dinu C., Ostrowski type inequalities on time scales, Annals of the University of Craiova-Mathematics and Computer Science Series, 34(2007), 43-58.

[14] Dragomir S.S., A generalization of Ostrowski integral inequality for mappings whose derivatives belong to $L_{1}[a, b]$ and applications in numerical integration, Journal of Computational Analysis and Applications, 3(4)(2001), 343-360.

[15] Dragomir S.S., A generalization of the Ostrowski integral inequality for mappings whose derivatives belong to $L_{p}[a, b]$ and applications in numerical integration, Journal of Mathematical Analysis and Applications, 255(2)(2001), 605-626.

[16] Dragomir S.S., Agarwal R.P., Cerone P., On Simpson's inequality and applications, RGMIA research report collection, 2(3)(1999).

[17] Dragomir S.S., Cerone P., Roumeliotis J., A new generalization of Ostrowski's integral inequality for mappings whose derivatives are bounded and applications in numerical integration and for special means, Applied Mathematics Letters, 13(1)(2000), 19-25.

[18] Feng Q., Meng F., Generalized Ostrowski type inequalities for multiple points on time scales involving functions of two independent variables, Journal of inequalities and applications, 2012(1)(2012), 74 pages.

[19] Hilger S., Ein makettenkalkül mit Anwendung auf Zentrumsmannigfaltigkeiten Ph. D, PhD thisis, thesis, 1988.

[20] Hilger S., Analysis on measure chains-a unified approach to continuous and discrete calculus, Results Math., 18(1-2)(1990), 18-56.

[21] Hilscher R., A time scales version of a Wirtinger-type inequality and applications, Journal of Computational and Applied Mathematics, 141(1)(2002), 219-226.

[22] Hussain S. Amer L.M., Alomari M., Generalized double-integral Ostrowski type inequalities on time scales, Applied Mathematics Letters, 24(8)(2011), 1461-1467.

[23] Kac V., Cheung P., Quantum calculus, Universitext, Springer-Verlag,, New York, 2002.

[24] Kermausuor S., Nwaeze, Eze R., Torres D.F.M., Generalized weighted Ostrowski and Ostrowski-Grüss type inequalities on time scale via a parameter function, Journal of Mathematical Inequalities, 11(4)(2017), 1185-1199.

[25] Li W.N., Some delay integral inequalities on time scales, Computers $\& 5$ Mathematics with Applications, 59(6)(2010), 1929-1936.

[26] Li W.N., Sheng W., Some Gronwall type inequalities on time scales, J. Math. Inequal., 4(1)(2010), 67-76.

[27] Li W.N., Sheng W., Some nonlinear dynamic inequalities on time scales, Proceedings Mathematical Sciences, 117(4)(2007), 545-554. 
[28] Liu W., Some weighted integral inequalities with a parameter and applications, Acta Applicandae Mathematicae, 109(2)(2010), 389-400.

[29] LiU W., Yu H., PAN X., New weighted Ostrowski-Grüss-Čebyšev type inequalities, Bulletin of the Korean Mathematical Society, 45(3)(2008), 477-483.

[30] Liu We., Xue Q., Wang S.-F., Several new perturbed Ostrowski-like type inequalities, J. Inequal. Pure Appl. Math., 8(4)(2007), pp. 6.

[31] LiU W., Several error inequalities for a quadrature formula with a parameter and applications, Computers $\&$ Mathematics with Applications, 56(7)(2008), 1766-1772.

[32] Liu W., Tuna A., Weighted Ostrowski, Trapezoid and Grüss type inequalities on time scales, J. Math. Inequal., 6(3)(2012), 381-399.

[33] LiU W., TunA A., Diamond- $\alpha$ weighted Ostrowski type and Grüss type inequalities on time scales, Applied Mathematics and Computation, 270(2015), 251-260.

[34] Liu W., Tuna A., JiAng Y., On weighted Ostrowski type, Trapezoid type, Grüss type and Ostrowski-Grüss like inequalities on time scales, Applicable Analysis, 93(3), (2014), 551-571.

[35] Mitrinović D.S., PeĈArić J., Fink A.M., Classical and New Inequalities in Analysis, volume 61, Springer Science \& Business Media, 2013.

[36] Mitrinović D.S., PeĈArić J., Fink A.M., Inequalities Involving Functions and Their Integrals and Derivatives, volume 53, Springer Science \& Business Media, 2012.

[37] NGô Q.A., LiU W.J., A sharp Grüss type inequality on time scales and application to the sharp Ostrowski-Grüss inequality, Commun. Math. Anal., $6(20)(2009), 33-41$.

[38] NwaEze Eze R., Generalized weighted trapezoid and Grüss type inequalities on time scales, Aust. J. Math. Anal. Appl., 14(1)(2017), 13.

[39] Nwazze Eze R., A new weighted Ostrowski type inequality on arbitrary time scale, Journal of King Saud University-Science, 29(2)(2017), 230-234.

[40] Nwaeze E.R., New integral inequalities on time scales with applications to the continuous and discrete calculus, Communications in Applied Analysis, $22(1)(2018), 1-17$.

[41] Nwaeze E.R., Kermausuor S., New Bounds of Ostrowski-Grüss type inequality for $(k+1)$ points on time scales, International Journal of Analysis and Applications, 15(2)(2017), 211-221.

[42] Nwaeze E.R., Kermausuor S., Tameru A.M., New time scale generalizations of the Ostrowski-Grüss type inequality for $k$ points, Journal of Inequalities and Applications, 201(245)(2017).

[43] Nwaeze E.R., TAmeru A.M., On weighted Montgomery identity for $k$ points and its associates on time scales, Abstract and Applied Analysis, (2017). Art. ID: 5234181 .

[44] Ostrowski A., Über die Absolutabweichung einer differentiierbaren Funktion von ihrem Integralmittelwert, Commentarii Mathematici Helvetici, 10(1)(1937), 226-227.

[45] SarIKaya M.Z., New weighted Ostrowski and Čebyšev type inequalities on time scales, Computers \& Mathematics with Applications, 60(5)(2010), 1510-1514. 
[46] Srivastava H.M., Tseng K.-L., Tseng S.-J., Lo J.-C., Some weighted Opial-type inequalities on time scales, Taiwanese Journal of Mathematics, (2010), 107-122.

[47] Tuna A., Daghan D., Generalization of Ostrowski and Ostrowski-Grüss type inequalities on time scales, Computers $\&$ Mathematics with Applications, $60(3)(2010), 803-811$.

[48] Tuna A., Jiang Y., Liu W., Weighted Ostrowski, Ostrowski-Grüss and Ostrowski-Čebyšev type inequalities on time scales, Publ. Math. Debrecen, 81(2012), 81-102.

[49] Yen C.-C., Ostrowski's inequality on time scales, Applied Mathematics Letters, 21(4)(2008), 404-409.

[50] Zheng B., Feng Q., Generalized dimensional Ostrowski type and Grüss type inequalities on time scales, Journal of Applied Mathematics, 2014(2014).

\author{
A. A. EL-DeEB \\ Department of Mathematics \\ FACULTY OF SCIENCE \\ Al-AzHAR UNIVERSITY \\ Nasr City (11884), CAIro, Egypt \\ e-mail: ahmedeldeeb@azhar.edu.eg \\ H. A. Elsennary \\ Department of Mathematics \\ FACUlty of EngineEring And Sciences \\ SinAi UnIVERSiTy \\ Arish (45615), North Sinai, Egypt \\ e-mail: hamza.abderabou@su.edu.eg \\ Eze R. Nwaeze \\ Department of Mathematics \\ Tuskegee UNIVERSITY \\ Tuskegee (36088), AL, USA \\ e-mail: enwaeze@tuskegee.edu
}

Received on 03.01.2018 and, in revised form, on 27.05.2018. 